\title{
Numerical analysis of subgrain formation during metal cutting and rolling based on the crystal plasticity theory
}

\author{
Fumihisa Nagashima' ${ }^{1}$ Yuki Nakagawa' ${ }^{1}$ Masahiko Yoshino ${ }^{1}[0$
}

Received: 12 February 2021 / Accepted: 10 January 2022 / Published online: 2 February 2022

(C) The Author(s) 2022

\begin{abstract}
The grain refinement technology is important in improving the metallic material properties without the requirement of additional alloy elements. Previously, we developed an efficient method for producing ultrafine-grained steel strips using a combination of cutting and heat treatment. However, the effect of cutting on recrystallization was not apparent. The objective of this study is to investigate the effects of metal cutting on static recrystallization and outline its advantages in grain refinement using numerical simulations based on the crystal plasticity theory. Simulation results show that shear deformation in metal cutting activates more slip systems than plane strain compression via rolling, even when considering the same equivalent plastic strain. The geometrically necessary dislocations are assumed to accumulate in the crystal because many slip systems are activated in shear deformation and improve grain refinement via static recrystallization in the subsequent heat treatment. This result indicates that the deformation type plays an important role in the recrystallization process. Thus, cutting is more efficient than rolling for the production of ultrafine-grained steel.
\end{abstract}

Keywords Crystal plasticity $\cdot$ Numerical simulation $\cdot$ Pure iron $\cdot$ Shear deformation $\cdot$ Subgrains $\cdot$ Static recrystallization

\section{Introduction}

The miniaturization of various industrial products has accelerated because of its many advantages, including the integration of functions as well as its space saving nature, improved portability, and resource and energy savings. Typical examples of such products include biomedical applications such as medical surgery devices (e.g., endoscopes), which enable minimally invasive treatment $[1,2]$. Many researchers have developed micromachining and microfabrication technologies to miniaturize such mechanical devices [3, 4]. The grain size of the component material exhibits considerable influence in the micromachining of the miniature components because the component size and volume of machining are almost the same as the grain size. The crystal grain size of structural steel sheets is greater than $10 \mu \mathrm{m}$, and this size is too large for microfabrication. Therefore, in re-cent

Masahiko Yoshino

yoshino.m.ab@m.titech.ac.jp

1 Department of Mechanical Engineering, Tokyo Institute of Technology, Tokyo, Japan years, ultrafine-grained steel comprising crystal grains of approximately $1 \mu \mathrm{m}$ in diameter has been attracting increasing research attention. Fine and accurately finished surfaces could be obtained using ultrafine-grained steel as work material for micromachining [5-7]. In addition, ultrafine-grained materials exhibit various advantages such as high yield stress [8], superplasticity [9], and resistance against stress corrosion cracking in stainless steel [10]. Because the properties of these materials can be improved without adding alloying elements, they exhibit excellent performance in terms of environmental measures such as material recyclability.

Ultrafine-grained steel is commercially produced via the thermomechanical control process (TMCP) [11, 12]. Deformation energy is applied and microstructural changes are induced via rolling in this process. Although high productivity can be achieved via rolling, the plastic strain is limited by the thickness of the products. Further, a large strain must be applied to the work material to produce ultrafine grains. In addition, the metallographic phenomena associated with TMCP, for example, dynamic transformation and recrystallization, are dependent on the chemical components. Thus, the optimal process conditions for each steel must be experimentally determined. 
Severe plastic deformation (SPD) methods have also been receiving increasing attention [13]. Several SPD methods, including equal-channel angular processing [14, 15], high-pressure torsion [16, 17], and accumulative rollbonding [18], have been proposed. These SPD methods can result in the accumulation of large plastic strains in the work material. This can be attributed to the fact that the initial shapes of the work materials are maintained even after repeated application of the SPD methods. Ultrafine grains of nanometer size can be produced using SPD methods. However, their productivity was low because they were batch processes.

An efficient process employing a metal cutting method was proposed for producing ultrafine-grained steel [19]. Because the chip generated via metal cutting is subjected to severe shear plastic deformation, a sufficient amount of plastic deformation energy is ac-cumulated in it. This energy allows the production of uniform ultrafine recrystallized grains when the chip is subjected to heat treatment. Unevenly sized grains tend to be generated when plastic deformation energy is applied to a material in a plate rolling process [20]. In addition, the average grain size obtained using the metal cutting process was smaller than that obtained via the rolling process, although a larger total equivalent plastic strain was applied in rolling. This is an interesting result because it reveals that grain refinement via recrystallization is dependent not only on the plastic work applied to the material [21-23] but also on the forming method.

In a previous paper [20], the disparity of recrystallized microstructure was attributed to the difference in the subgrain structure of deformed grains in case of the chip produced via metal cutting and the plate produced via rolling. Plastic deformation occurs in a shear plane with a thickness lower than the diameter of the grain in the metal cutting process, and all the grains are deformed equivalently by severe shear plastic strain. Thus, uniform subgrains are generated in all the grains of the chip specimen, causing uniform recrystallization during the subsequent heat treatment. Further, during the rolling process, plastic deformation occurs between two rollers, where the deformation zone volume is considerably larger than the grain volume. Uneven plastic strain can be observed with respect to the grains in the deformation zone because the plastic deformation of grains is dependent on the initial crystal orientation. Thus, uneven subgrains are generated, and uneven recrystallization occurs. However, the effect of the forming process on the development of the subgrain structure and subsequent recrystallization was not apparent previously. The difference in the development of the subgrain structure was assumed to be dependent not only on the difference in the size of the plastic deformation zone but also on the difference in deformation type, i.e., shear deformation in cutting and plane strain compression in rolling.
Thus, herein, the effect of the deformation type on the crystal plastic deformation of grains is studied numerically based on the crystal plasticity theory. More slip systems are activated via shear deformation in the metal cutting process when compared with those obtained via plane strain compression by rolling even though their equivalent plastic strains are identical. The difference between the activated slip systems influences the sub-grain structure and subsequent recrystallization. In addition, the advantages of the metal cutting method with respect to the production of ultrafine-grained steel are discussed.

\section{Experimental research}

\section{Material and methods}

A commercial polycrystalline pure iron rod was used as the test material for the orthogonal cutting and cold plate rolling experiments. Details of the experimental method have been reported elsewhere [20]. In orthogonal cutting, a chip is obtained via severe shear deformation on the shear plane, as shown in Fig. 1a. The metal-cutting process is idealized by assuming only a single shear strain component associated with the chip. This shear strain can be calculated as follows [24]:

$\gamma=\cot \phi+\tan (\phi-\alpha)$

where $\alpha$ is the rake angle of the cutting tool and $\phi$ is the shear angle.

$\tan \phi=\frac{\left(t_{0} / t_{c}\right) \cos \alpha}{1-\left(t_{0} / t_{c}\right) \sin \alpha}$,

where $t_{0}$ is the depth of the cut and $t_{c}$ is the chip thickness. The equivalent plastic strain can be calculated by integrating the following equation.

$\mathrm{d} \bar{\varepsilon}=\sqrt{\frac{2}{3}} \sqrt{\mathrm{d} \varepsilon_{x x}^{2}+\mathrm{d} \varepsilon_{y y}^{2}+\mathrm{d} \varepsilon_{z z}^{2}+2\left(\mathrm{~d} \varepsilon_{x y}^{2}+\mathrm{d} \varepsilon_{y z}^{2}+\mathrm{d} \varepsilon_{z x}^{2}\right)}$

where $\varepsilon_{x x}, \varepsilon_{y y}$, and $\varepsilon_{z z}$ are the normal strain elements and $\varepsilon_{x y}$, $\varepsilon_{y z}$, and $\varepsilon_{z x}$ are the shear strain elements of the strain tensor. The total plastic strain applied via orthogonal cutting can be obtained by assuming simple shear deformation.

$\bar{\varepsilon}_{c}=\int \sqrt{\frac{2}{3}} \sqrt{0+0+0+2\left(\mathrm{~d} \varepsilon_{x y}^{2}+0+0\right)}=\frac{2}{\sqrt{3}} \varepsilon_{x y}=\frac{\gamma}{\sqrt{3}}$

The cutting conditions and shear strain applied in case of the chip are presented in Table 1. 
(a)

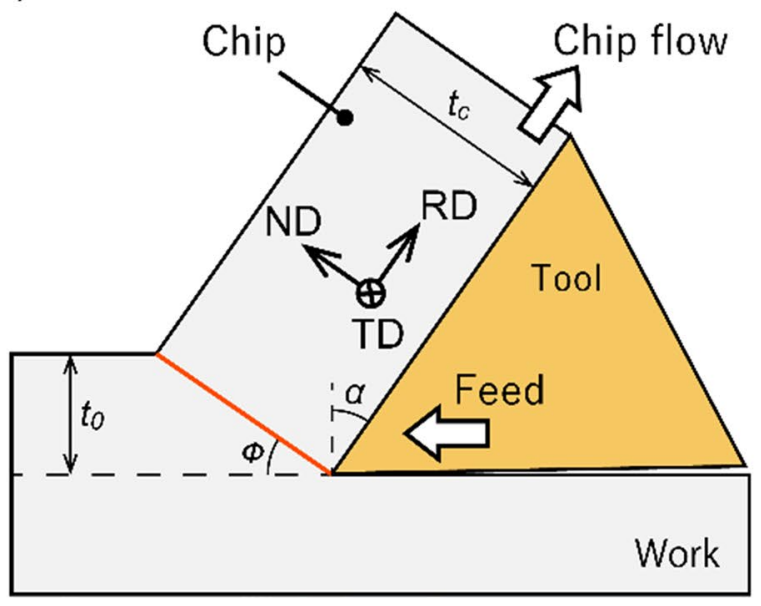

Fig. 1 Schematic of (a) orthogonal cutting and (b) plate rolling
Table 1 Cutting conditions and chip geometry [20]

\begin{tabular}{ll}
\hline Parameter & Condition \\
\hline Tool material & Carbide \\
Rake angle $\alpha$ & $10^{\circ}$ \\
Cutting speed $V$ & $25 \mathrm{~m} / \mathrm{min}$ \\
Depth of cut $t_{0}$ & $0.04 \mathrm{~mm}$ \\
Lubrication & Dry \\
Chip thickness $t_{c}$ & $0.15 \mathrm{~mm}$ \\
Shear angle $\phi$ & $15.8^{\circ}$ \\
Shear stain $\gamma$ & 3.6 \\
Equivalent strain $\bar{\varepsilon}_{c}$ & 2.1 \\
\hline
\end{tabular}

For the plate rolling experiment, rectangular samples were cut from the same iron rod used for the cutting experiment via wire electric discharge machining. The samples were compressed by $91 \%$ via single-pass rolling, as shown in Fig. 1b. The equivalent plastic strain applied by plate rolling can be obtained by assuming plane strain compression. (b)

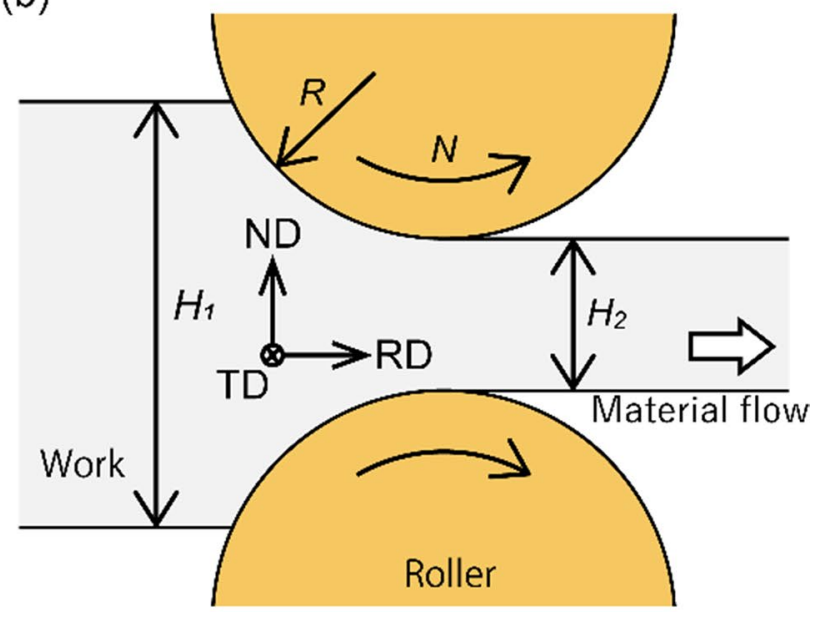

Table 2 Rolling conditions and geometry of the rolled plate [20]

\begin{tabular}{ll}
\hline Parameter & Condition \\
\hline Roll diameter $R$ & $40 \mathrm{~mm}$ \\
Rolling speed $V_{R}$ & $10 \mathrm{rpm}$ \\
Thickness reduction rate $\frac{H_{1}-H_{2}}{H_{1}}$ & 0.91 \\
Number of rolling passes & 1 \\
Lubrication & Dry \\
Thickness before rolling $H_{1}$ & $2.96 \mathrm{~mm}$ \\
Thickness after rolling $H_{2}$ & $0.27 \mathrm{~mm}$ \\
Logarithm strain $\ln \left(\frac{H_{1}}{H_{2}}\right)$ & 2.39 \\
Equivalent strain $\bar{\varepsilon}_{r}$ & 2.76 \\
\hline
\end{tabular}

of the deformed and recrystallized microstructures were analyzed using the electron backscatter diffraction (EBSD)

$\bar{\varepsilon}_{r}=\int \sqrt{\frac{2}{3}} \sqrt{\mathrm{d} \varepsilon_{x x}^{2}+\mathrm{d} \varepsilon_{y y}^{2}+0+2(0+0+0)}=\frac{2}{\sqrt{3}} \varepsilon_{x x}=\frac{2}{\sqrt{3}} \ln \left(\frac{H_{1}}{H_{2}}\right)$

where $H_{1}$ and $H_{2}$ are the plate thicknesses before and after rolling, respectively. The rolling conditions and equivalent strain applied in the rolling process are presented in Table 2.

After cutting or rolling, the samples were subjected to heat treatment under an argon gas atmosphere to induce static recrystallization. The temperature was set as $500{ }^{\circ} \mathrm{C}$ and $600{ }^{\circ} \mathrm{C}$, and the samples were heated for $30 \mathrm{~s}, 1 \mathrm{~min}$, and $5 \mathrm{~min}$. Then, the samples were quickly cooled by blowing argon gas. The crystal orientation distributions method. The reference direction (RD)-normal direction (ND)-transverse direction (TD) coordinate system used for EBSD analysis is presented in Fig. 1. RD corresponded to the chip flow direction, ND corresponded to the thickness direction of the chip, and TD corresponded to the chip width direction in case of the chip samples (Fig. 1a). RD corresponded to the rolling direction, ND corresponded to the normal direction of the rolled surface, and TD corresponded to the width direction in case of the rolled samples (Fig. 1b). 


\section{Experimental results}

Figures 2 and 3 present the microstructures of the chip and rolled plate, respectively. Figures $2 \mathrm{a}$ and $3 \mathrm{a}$ show the inverse pole figure (IPF) maps of the deformed samples. Figures $2 b$ and $3 \mathrm{~b}$ present the kernel average misorientation (KAM) maps of the deformed samples. Finally, Figs. $2 \mathrm{c}$ and $3 \mathrm{c}$ provide the IPF maps of the recrystallized samples. A uniform ultrafine-grained microstructure with a diameter of $0.2 \mu \mathrm{m}$ was observed to be generated in the chip (Fig. 2a). However, the grain size in case of the rolled plate (Fig. 3a) was not uniform.

Based on the KAM map of the chip samples (Fig. 2b), the misorientation of the crystal lattice was observed to be concentrated and formed grain boundaries. These boundaries are indicated by black lines, and the misorientation angle was greater than $5^{\circ}$. Thus, the misorientation inside the grains remained low, and majority of the area in Fig. $2 b$ was blue. In contrast, a low misorientation region could be observed inside the grains in case of the KAM map of the rolled samples (Fig. 3b). For misorientation angles smaller than $5^{\circ}$, the grains were represented by green or yellow colors. However, they were not concentrated and did not form grain boundaries. Therefore, many subgrain boundaries were generated, and the grains were uniformly subdivided into ultrafine grains in the chip samples. Further, coarse and heterogeneous subgrain structures were observed in the rolled plate.

Based on the IPF map of the chip samples obtained after heat treatment (Fig. 2c), fine recrystallized grains were observed to be generated. The size of the recrystallized grains was almost uniform. Grains of various sizes were observed based on the IPF map of the rolled samples after heat treatment (Fig. 3c), and uneven microstructures (a)

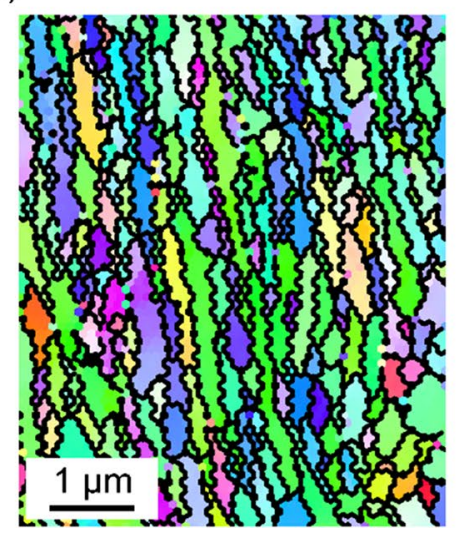

(b)

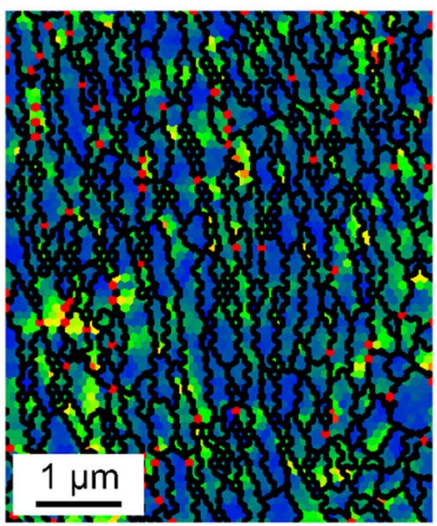

(c)

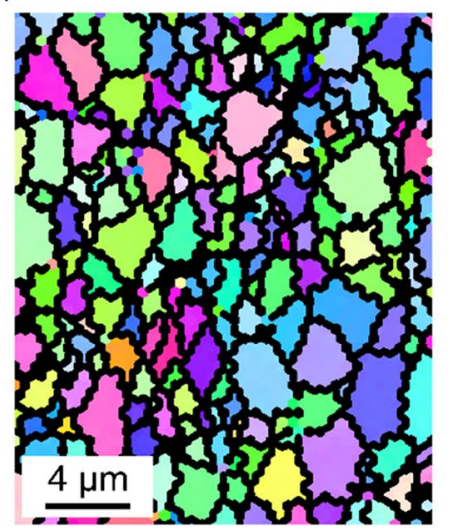

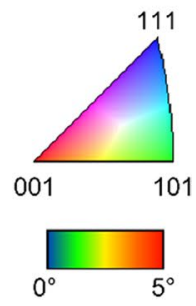

ND

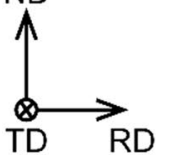

Fig. 2 Microstructure of the chip. a Inverse pole figure (IPF) map for transverse direction (TD), b kernel average misorientation (KAM) map of the deformed microstructure, and $\mathbf{c}$ IPF map for TD of the recrystallized microstructure after heat treatment at $500{ }^{\circ} \mathrm{C}$ for $5 \mathrm{~min}$

(a)

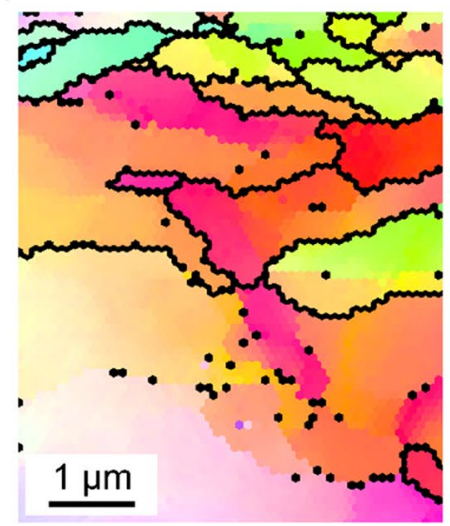

(b)

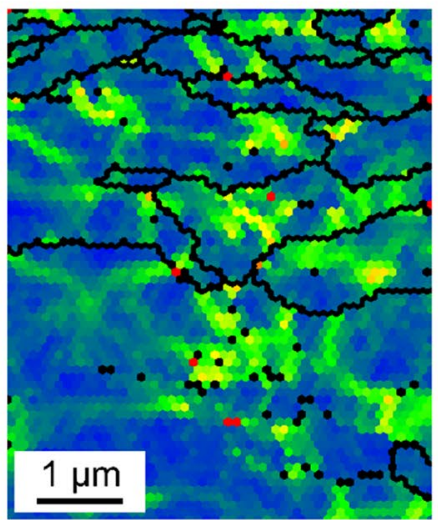

(c)

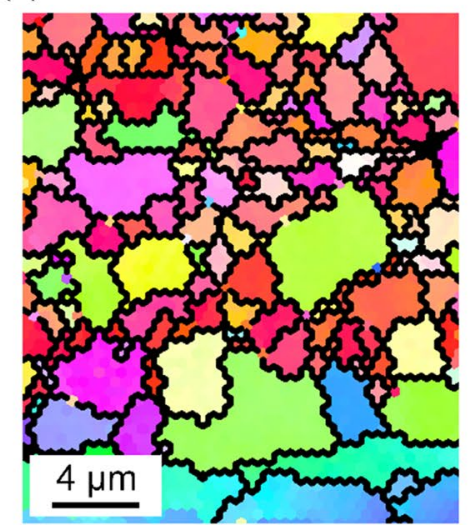

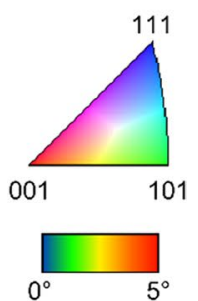

ND

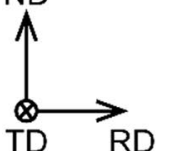

Fig. 3 Microstructure of the rolled plate. a IPF map for TD, b KAM map of the deformed microstructure, and $\mathbf{c}$ IPF map for TD of the recrystallized microstructure after heat treatment at $500{ }^{\circ} \mathrm{C}$ for $5 \mathrm{~min}$ 
were obtained. The differences in the recrystallized microstructures can be attributed to the variations in the subgrain structures.

The subgrains of the chip specimen in Fig. 2a were smaller and more uniform than the subgrains of the rolled specimen in Fig. 3a although the equivalent plastic strain of the chip specimen $\left(\bar{\varepsilon}_{c}=2.1\right)$ was smaller than the rolled specimen $\left(\bar{\varepsilon}_{c}=2.76\right)$. This indicates that the cutting process is more efficient at storing plastic strain to generate subgrains than the rolling process. Therefore, the chip specimens generate more nucleation sites for recrystallization.

\section{Effects of deformation type on generated subgrain structure}

In many studies discussing microstructure control technologies $[22,25]$, the effect of plastic deformation on the recrystallization process has been evaluated using the equivalent plastic strain. For example, the recrystallization rate was expressed using a model formula based on the equivalent plastic strain in the case of the microstructure simulation system developed by Dupin et al. [26]. However, the relationship between the orientation of the crystal grains and the plastic strain component has a significant influence on the subgrain generation because the deformation of grains depends on the anisotropy of the crystal. Thus, the generated subgrain structure is influenced not only by the equivalent strain but also by the type of deformation process. The differences in subgrain structures between the chip and rolled specimens are caused by three mechanisms; (1) differences in deformation zone size, (2) effects of material property changes in the processes, and (3) completely different applied deformation.

Regarding (1), it was considered that the deformation boundary conditions of crystal grains in the work material was very different as shown in Fig. 4 owing to difference in size of plastic deformation zone between cutting and rolling [20]. Grains were deformed in a thin shear plane in case of cutting. The thickness of the shear deformation zone can be evaluated as follows. Because the thickness of the shear plane could be assumed to be approximately $1 / 6$ of the length of the shear plane [27], we obtain

$$
\begin{gathered}
\frac{t_{0}}{\delta \sin \phi}=6 \\
\delta \approx 24 \mu \mathrm{m}
\end{gathered}
$$

The thickness of the shear plane was smaller than the grain size as shown in Fig. 4a because the average grain

(a)

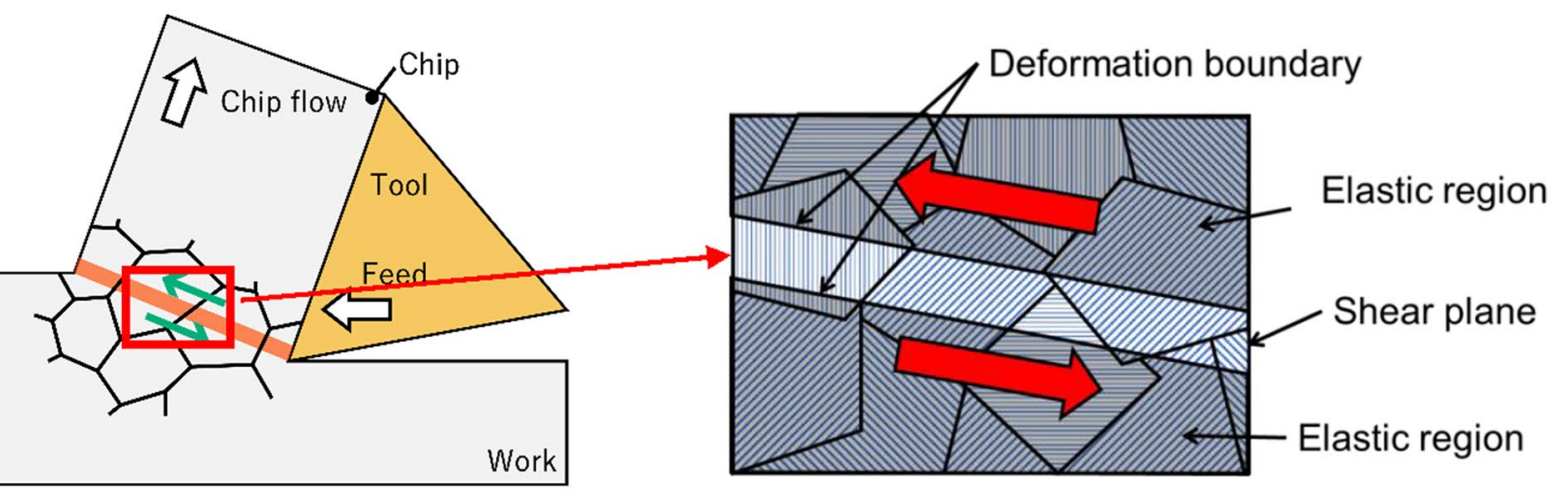

(b)
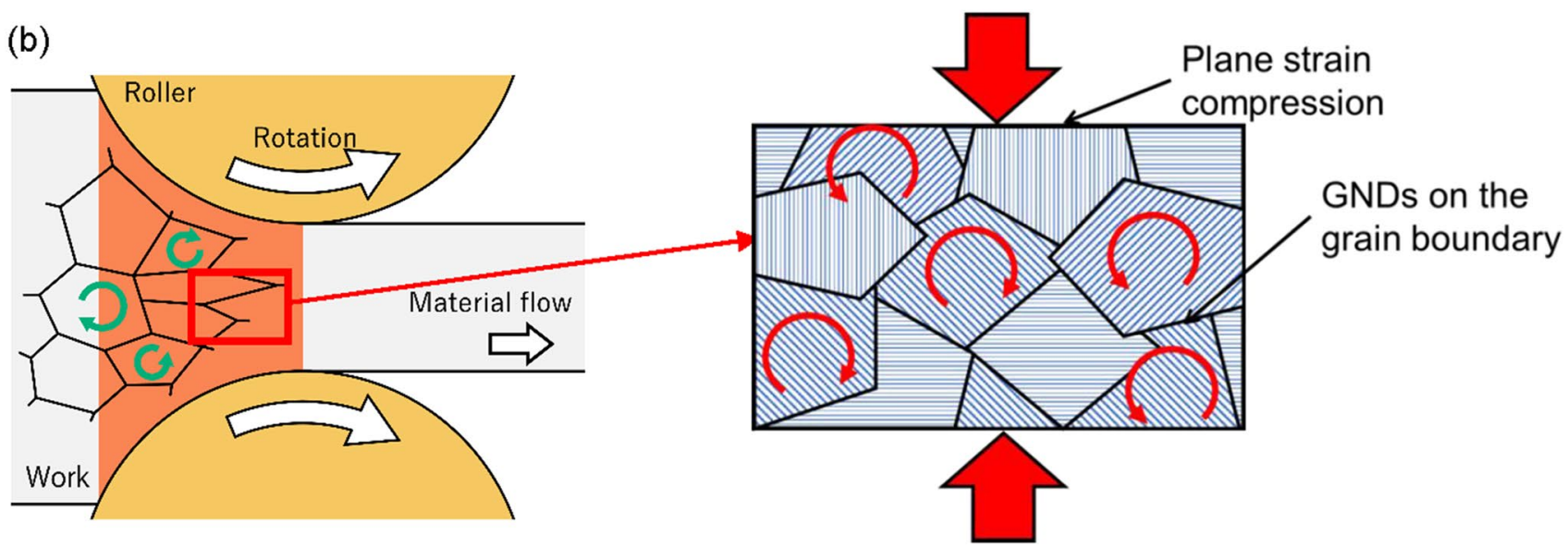

Fig. 4 Plastic deformation zone of $\mathbf{a}$ cutting and $\mathbf{b}$ rolling 
diameter of the work material was $83 \mu \mathrm{m}$. The upper and lower boundaries of the shear plane served as the restricting boundaries of the plastic deformation zone because the exterior parts of the shear plane were elastic. Therefore, when the grains passed through the shear plane, they all deformed evenly. In the deformation zone, there were few grain boundaries, and strain relaxation on the grain boundary rarely occurred. All plastic deformation energy was consumed in the grains crystal and accumulated in the dislocations, and uniform ultrafine subgrains were generated in the grains.

Conversely, as shown in Fig. 4b, the deformation zone in the rolling process was between the rollers that were in contact with the work material. The contact length $\mathrm{L}$ between a roller and the material is calculated as follows using the data in Table 2.

$L=\sqrt{\left(H_{1}-H_{2}\right) R-\frac{\left(H_{1}-H_{2}\right)^{2}}{4}}=7.21 \mathrm{~mm}$

The height of the plastic deformation zone comprised the plate thickness between the rolls, and it was ranged from 0.27 to $2.96 \mathrm{~mm}$ as shown in Table 2. Because the average crystal grain size of the work material was approximately $83 \mu \mathrm{m}$, the plastic deformation zone was considerably larger than the crystal grains in the material (Fig. 4b). Therefore, the crystal grains in the material were deformed by the stress exerted by the adjacent crystal grains. The grains inside the deformation zone exhibited different crystal orientations, and their hardness differed with respect to the compressive stress in the thickness direction. The grains with "soft" crystal orientation deformed first, whereas those with "hard" crystal orientation deformed later. As a result, dislocations accumulated on the grain boundaries, strain mismatch and sliding occurred owing to plastic deformation [28]. Due to the mismatch and sliding, the grains were rotated individually to a certain crystal orientation at which they were easily deformed, resulting in a rolled texture [29]. Therefore, the subgrain structure in the rolled samples became uneven.

Engler et al. [30] developed a crystal-plastic finite element method based on the Grain Interaction (GIA) model, which incorporates deformation inconsistencies at grain boundaries, simulating the texture development of $\mathrm{Al}$ alloys through plate rolling. In the GIA model, the total crystal deformation energy is evaluated in the following equation, and the deformation is calculated to minimize the deformation energy.

$E_{\text {tot }}=\sum_{n=1}^{8} \sum_{S_{n}}\left(\tau_{n}\left|\gamma_{n}^{s}\right|\right)+\sum_{G B s, I F s}\left(E_{i j}^{G N D^{B}}+E_{i i}^{G N D^{B}}\right)$

where the first term on the right side is the deformation energy of the slip systems in a crystal lattice and the second term on the right side is the energy to generate geometrically necessary dislocations (GND) that compensates for the deformation mismatch on the grain boundaries. The tensor index ij represents the shear component, and ii represents the elongation component. The following equation expresses the energy of GND.

$E_{i j}^{G N D^{B}}=\frac{G b^{2}}{2} \rho_{i j}^{B}$

where $\mathrm{G}$ is the shear elastic modulus and $\mathrm{b}$ is the Burgers vector. $\rho_{i j}^{B}$ is the density of GND and is expressed by the following equation.

$\rho_{i j}^{B}=\frac{2\left|\Delta d \varepsilon_{i j}^{B}\right|}{b d_{k}}$

where $d_{k}$ is the grain size and $\Delta d \varepsilon_{i j}^{B}$ is the shear strain difference along the grain boundaries. According to Engler et al., this simulation can describe the formation of the $\mathrm{Al}$ alloy's texture.

In the GIA model, part of the deformation energy is consumed for generating GND at the grain boundaries, which reduced the deformation energy in the crystal lattice and suppressed the dislocation accumulation. The same mechanism in the rolling experiment conducted herein reduced the slip deformation in the crystal and suppressed the generation of subgrains. Similar analysis can be used to compare the activity of dislocations in crystal grains during pure iron rolling, but the quantitative comparison is unsuitable because some parameters should be assumed in this simulation. However, slip on the grain boundaries reduces the plastic strain in the crystal, which also reduces the dislocation density. This mechanism resulted in an uneven and coarse subgrain structure.

Regarding (2) effects of material property changes in the processes, critical shear stresses of slip systems in the grains differed because of the difference in strain rate and temperature between cutting and rolling, and this difference caused complicated influences on the subgrain structure.

The shear strain rate on the shear plane was calculated using the two-dimensional cutting theory as follows:

$\dot{\gamma}_{s}=\frac{V_{s}}{\delta}=\frac{\gamma_{s}}{\delta} \sin \varphi V$

Under the cutting conditions in Table 1, it was expressed as

$\dot{\gamma}_{s}=\frac{3.6 \sin 15.8^{\circ}}{24 \times 10^{-3}} \times \frac{25 \times 10^{3}}{60} \cong 17000 \mathrm{~s}^{-1}$

This was converted to the equivalent plastic strain rate as follows: 
$\bar{\varepsilon}_{c}=\frac{\dot{\gamma}_{s}}{\sqrt{3}} \cong 9800 \mathrm{sec}^{-1}$

However, in plate rolling, the strain rate was calculated as follows:

$\dot{\bar{\varepsilon}}_{r}=\frac{\bar{\varepsilon}_{r}}{d t}=\bar{\varepsilon}_{r} \frac{V_{r}}{L}$

Under the rolling conditions in Table 2, it was expressed as

$\dot{\bar{\varepsilon}}_{r}=2.76 \times \frac{20.9}{7.21}=8.03 \mathrm{~s}^{-1}$

The strain rate of rolling was approximately $1 / 1000$ of cutting. As the mobility of dislocations in a crystal depends on the strain rate, the critical shear stress $\tau_{c}$ of each slip system in the crystal increases as the strain rate increases. Thus, the strain rate of each slip system differed, as did the dislocation structures generated by cutting and rolling.

Meanwhile, the cutting and the rolling experiments were conducted under room temperature conditions, and the specimen temperature was not controlled. Strong shear strain occurred in the cutting experiment on a thin shear plane, where the strain rate is approximately 1000 times that of rolling. Thus, heat was concentrated on the shear plane, and the temperature quickly increased. Figure 5 shows a thermographic image of the chips used in the cutting experiment; the chip temperature was shown to rise to approximately $190{ }^{\circ} \mathrm{C}$. In steel cutting, many studies reported much higher chip temperatures [31, 32]. It is difficult to estimate the accurate chip temperature of the cutting experiment because the cutting temperature depends on the cutting conditions and the work material. However, temperature distribution in the thickness direction can be neglected because it becomes

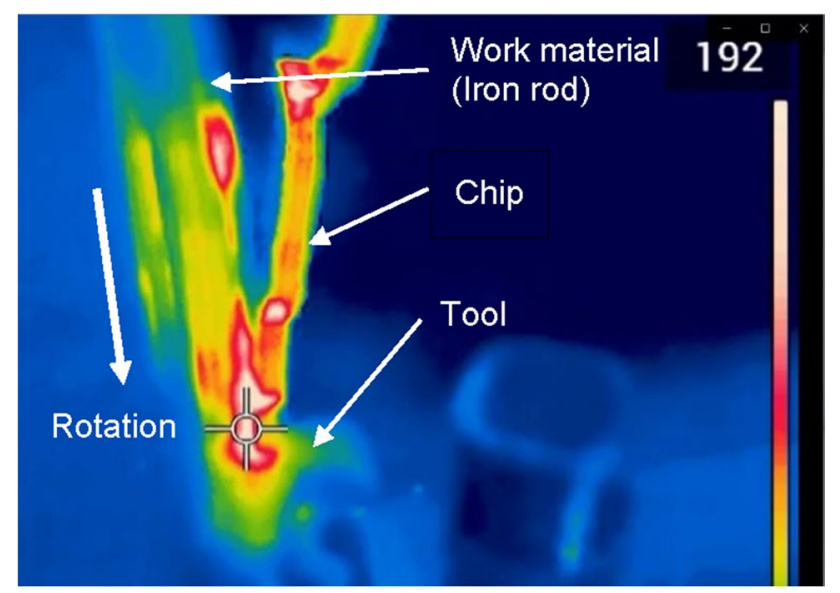

Fig. 5 Thermography image of a chip and work material in cutting experiment with a lathe uniform immediately (see appendix 1). Thus, crystal grains in the chip specimen were subjected to almost uniform thermal effect.

Conversely, in rolling, the contact area of a specimen to the rollers were relatively large and the deformation speed was comparably low. The heat generated by plastic deformation did not concentrate in the deformation zone, and easily dissipated to the rollers. Temperature rise in rolling was estimated as below when thermal dissipation and temperature distribution were neglected,

$\Delta T=\frac{W}{\rho C_{v}}=\frac{\bar{\sigma} \bar{\varepsilon}_{r}}{\rho C_{v}}=\frac{200 \times 2.76 \times 10^{6}}{7800 \times 461}=153^{\circ} \mathrm{C}$

where the density of iron $\rho=7800\left[\mathrm{~kg} / \mathrm{m}^{3}\right]$, the specific heat $C_{v}=461\left[\mathrm{~J} / \mathrm{kg}^{\circ} \mathrm{C}\right]$ and flow stress $\bar{\sigma}=200[\mathrm{MPa}]$ is. were obtained from literatures. However, the actual temperature rise in the rolling experiment is less than $153^{\circ} \mathrm{C}$ because the thermal dissipation is neglected in this calculation.

Temperature rise causes thermal softening of the material. This is because dislocation mobility increases as thermal energy increases, and the critical shear stress of each slip system decreases. Temperature rise also causes recovery of the strain hardening. Temperature change and strain rate influenced the accumulation of dislocation in the crystal grains and caused a difference in subgrain structures between the chip and rolled specimens. However, it is difficult to discuss their quantitative effect. Further study is necessary to reveal their mechanisms.

\section{Numerical simulation for demonstrating the effect of deformation type on subgrain formation}

Regarding (3) completely different applied deformation, it is considered that the disparity of microstructure was also dependent on the deformation type, including compressive and shear deformations, and caused the activation of different slip systems. It is difficult to investigate the effect of deformation type on the crystal orientation and dislocation multiplication based on the experimental results. Therefore, the effect of the deformation type on slip system activation in each grain was studied numerically based on the crystal plasticity theory. A single cubic element was used to avoid the effect of sliding on grain boundaries and investigate the effect of the deformation type. Slip system activation was simulated by applying plane strain compressive deformation and simple shear deformation.

The constitutive model used in this study follows the continuum mechanical framework of elastoplasticity 
established by Asaro [33] and Peirce et al. [34]. Detail of the framework is explained in the appendix 2.

$\dot{\gamma}^{\alpha}=\dot{\gamma}_{0}\left(\frac{\left|\tau^{\alpha}\right|}{\tau_{c}}\right)^{\frac{1}{m}} \operatorname{sgn}\left(\tau^{\alpha}\right)$

where $\dot{\gamma}^{\alpha}$ and $\tau^{\alpha}$ are the shear rate and resolved shear stress on the $\alpha$-th slip system, respectively, $\dot{\gamma}_{0}$ is the reference shear rate, $\tau_{c}$ is the critical resolved shear stress, and $m$ is the strain rate sensitivity index. The isotropic hardening system proposed by Kocks and Mecking [35] was employed in this model. All the slip systems were assumed to harden equivalently with the hardening law that governs the evolution of the critical resolved shear stress [36].

$\dot{\tau}_{\mathrm{c}}=h_{0}\left(\frac{\tau_{s}-\tau_{c}}{\tau_{s}-\tau_{0}}\right) \sum_{\alpha}\left|\dot{\gamma}^{\alpha}\right|$

where $h_{0}$ is the initial hardening rate, $\tau_{s}$ is the saturation critical resolved shear stress, and $\tau_{0}$ is the initial critical resolved shear stress.

Table 3 Material parameters in the crystal plasticity model

\begin{tabular}{lll}
\hline Type & Parameter & Value \\
\hline Elastic stiffness $^{* 1}$ & $c_{11}, c_{12}, c_{44}$ & $228 \mathrm{GPa}$, \\
& & $132 \mathrm{GPa}$, \\
$\{110\}$ slip systems $^{* 2}$ & $m$ & $116.5 \mathrm{GPa}$ \\
& $\dot{\gamma}_{0}$ & 0.05 \\
& $h_{0}$ & 0.01 \\
& $\tau_{0}$ & $150.5 \mathrm{MPa}$ \\
$\{112\}$ slip systems & & $40.0 \mathrm{MPa}$ \\
& $\tau_{s}$ & $250.875 \mathrm{MPa}$ \\
& $m$ & 0.05 \\
& $\dot{\gamma}_{0}$ & 0.01 \\
& $h_{0}$ & $67.5 \mathrm{MPa}$ \\
& $\tau_{0}$ & $58.0 \mathrm{MPa}$ \\
& $\tau_{s}$ & $250.0 \mathrm{MPa}$ \\
\hline
\end{tabular}

*1: Reported by Lord and Beshers [36], *2: Reported by Luo et al. [35]

Fig. 6 Single-crystal cubic element for crystal plasticity simulation: a plane strain compressive deformation and $\mathbf{b}$ simple shear deformation
The material is pure iron, which exhibits a body-centered cubic lattice crystal structure. Twenty-four slip systems of $\{110\}\langle\overline{1} 11\rangle$ and $\{112\}\langle 11 \overline{1}\rangle$ were used in the simulation. All the parameters used are presented in Table 3. The parameters associated with each system were experimentally determined [37], and the elastic stiffness coefficients were obtained from literature [38].

Figure 6 presents the numerical single-crystal cubic model. Figure 6a presents the plane strain compression of rolling, and Fig. 6b presents the simple shear deformation of cutting. The simulation was conducted on 3000 random crystal orientations. The deformation rate was determined based on the strain rate in the experiments.

Figure 7 compares the pole figures obtained numerically and experimentally to evaluate the validity of the crystal rotation model. The simulation of shear deformation represents variations in the crystal lattice orientation via cutting and that of plane strain compression represents variations in the crystal lattice orientation via rolling. The total strains of the simulation were set to correspond with those obtained based on experimental data; shear strain $\gamma=3.6$ for shear deformation and thickness reduction $=91 \%$ for plane strain compression. The simulated pole figures of the deformed microstructures were similar to those obtained experimentally. Fig. 7a and $b$ show the concentration of the $\{110\}$ planes. Moreover, they show similar concentration in a band area including $\{111\}$ planes. Fig. 7c and d show the concentration of the $\{122\}$ planes, and $\{102\}$ planes. Some differences in distribution are observed in Fig. 7c and d; however, they might be attributed to the slip deformation on the grain boundaries discussed in the section 2.3. Because this simulation used a simple model of a unit cubic cell and it did not involve effects of indetermined factors, such as slide on the grain boundary and friction with a tool, the results indicate fundamental characteristics of plastic deformation of the crystal lattices. Thus, these simulation results can be used to evaluate the activity of the slip systems of the crystal lattice during cutting and rolling, when the effect of slide deformation on the grain boundaries is neglected. Although, this simulation does not correspond quantitatively with the practical deformation of a grain, it can (a)

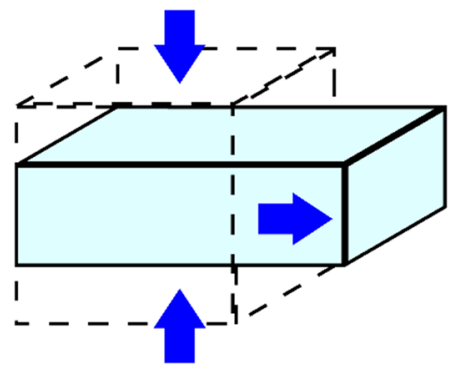

(b)

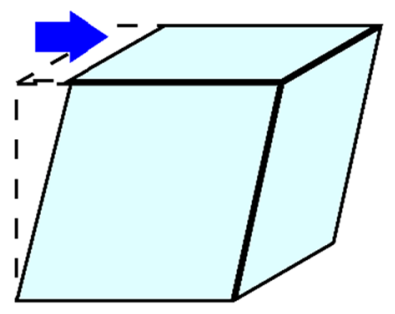

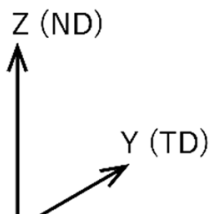

$X(R D)$ 


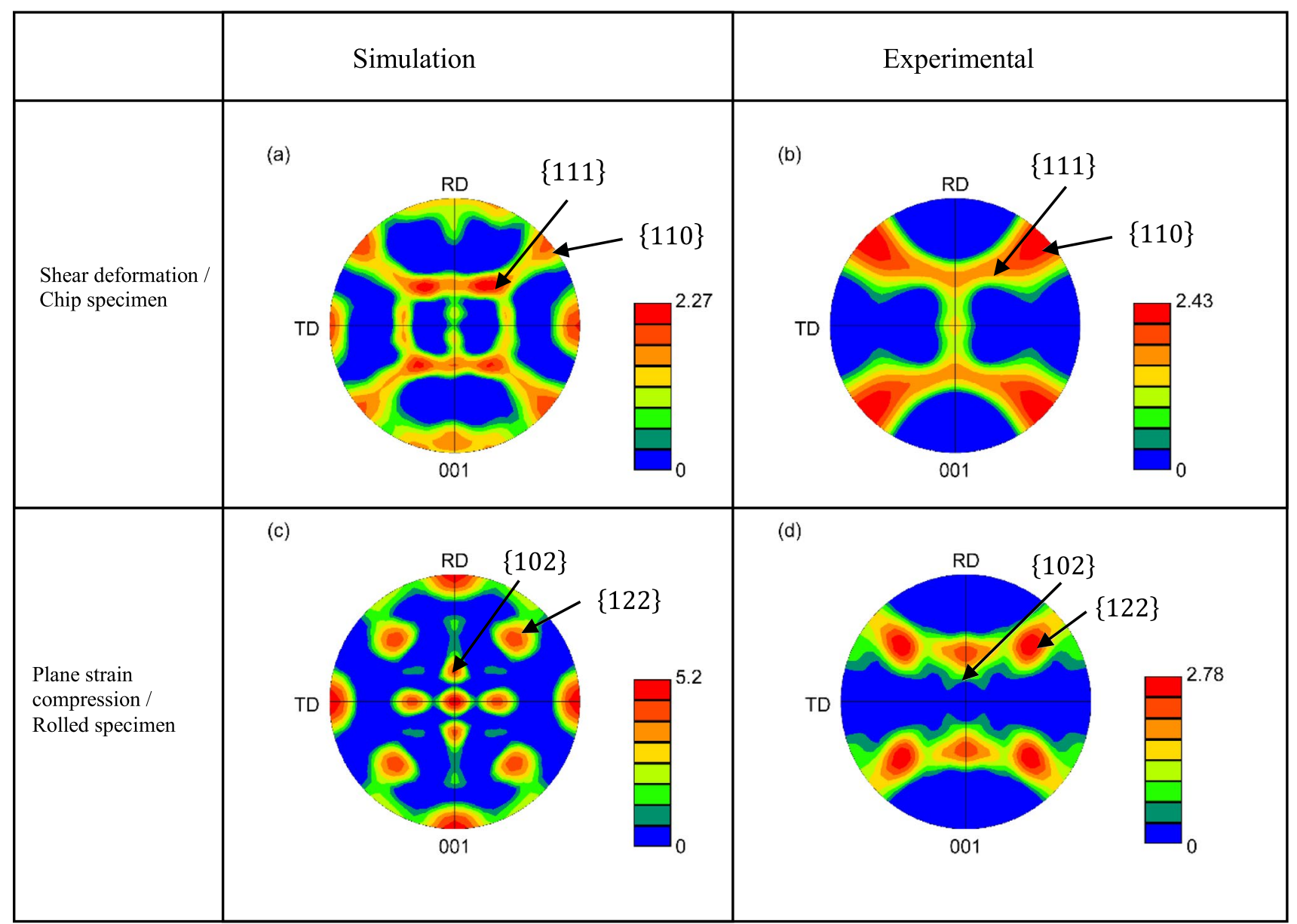

Fig. 7 Comparison of the (001) pole figures for simulation and experiment: a simulated pole figure of shear deformation, b pole figure of the chip samples, $\mathbf{c}$ simulated pole figure of plane strain deformation, and $\mathbf{d}$ pole figure of the rolled samples

be used to qualitatively evaluate the effect of plastic deformation on subgrain formation based on the simulation of the dislocation activity in the crystal.

A slip system whose resolved shear stress exceeds $98 \%$ of the critical shear stress is referred to as the active slip system. Its shear stress is governed by the following relation.

$\left|\dot{\gamma}_{\text {active }}\right| \geq \dot{\gamma}_{0}\left(\frac{0.98 \tau_{c}}{\tau_{c}}\right)^{\frac{1}{m}}$
$=6.68 \times 10^{-3}$

Thus, slip systems that satisfy eq. (20) are considered to be active slip systems throughout the deformation process. $t_{\text {total }}$ indicates the total deformation time.

$\left|\gamma_{\text {active }}\right|=\left|\dot{\gamma}_{\text {active }}\right| t_{\text {total }}$
$\quad \geq 6.68 \times 10^{-3} t_{\text {total }}$

For numerical simulation, we considered 24 active slip systems satisfying eq. (20). The deformation parameters used in the simulation are presented in Table 4.
Figure 8 indicates the fraction of crystal orientations versus the number of activated slip systems in each crystal lattice. Numerical results were obtained for shear deformation and plane strain compression when considering 3000 random initial orientations. The number of activated slip systems obtained via plane strain compressive deformations was less than nine for majority of the crystal orientations. However, more than ten activated slip systems could be obtained via shear deformations for majority of the crystal orientations. This result indicates that the probability of

Table 4 Simulation conditions used for obtaining activated slip systems

\begin{tabular}{lll}
\hline Parameter & Shear deformation & Plane strain deformation \\
\hline Equivalent strain & 2.8 & 0.01 \\
Deformation & $\gamma=4.8$ & $\mathrm{H}_{2} / \mathrm{H}_{1}=0.09$ \\
Deformation rate & $2.84 \times 10^{4}$ & 2.58 \\
Deformation time $t_{\text {total }}$ & $1.69 \times 10^{-4}$ & 0.352 \\
\hline
\end{tabular}




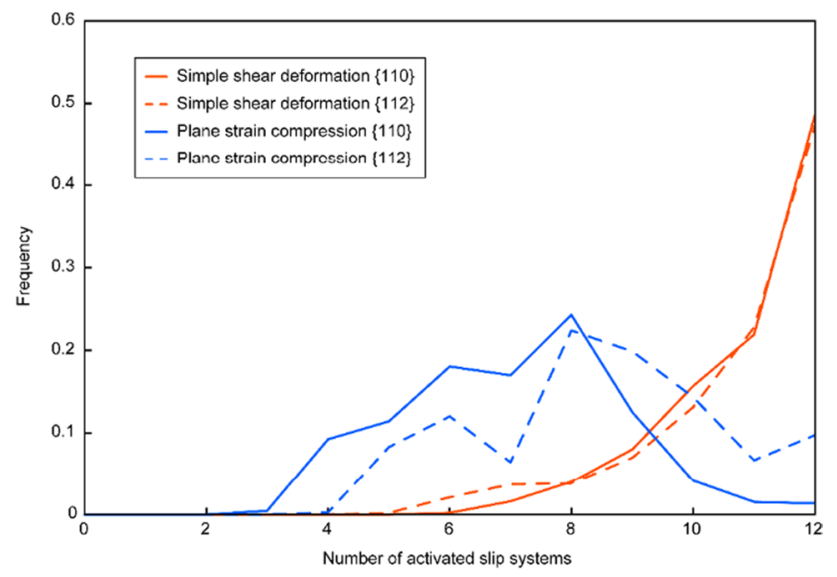

Fig. 8 The number of active slip systems in simulation

slip system activation is higher for shear deformation when considering identical total equivalent plastic strains. Misorientations inside the grains are supposed to be generated more because of the multiple activations of slip systems in shear deformation when compared with that in plane strain compression. These misorientations transform into geometrically necessary dislocations and form geometrically necessary boundaries (GNBs). Thus, many ultrafine subgrains are generated in the crystal. These subgrains are considered as the nuclei of recrystallization. Therefore, when applying the same equivalent plastic strain to the material, more subgrains are generated via shear deformation than via plane strain compression.

Shear strain deformation occurs during the practical cutting process, and the plane strain compressive deformation occurs during the practical rolling process. Thus, dislocations are accumulated more in the cutting process than in the rolling process owing to the difference in deformation type. By the addition of the effect of the mechanism of (1), smaller subgrains are uniformly generated in the chip specimen than in the rolled specimen. They provide many nucleation sites and generate fine recrystallized grains when the specimen is applied to following heat treatment. Difference in the deformation type has an apparent influence on the development of subgrains and recrystallized grain structures. Cutting is advantageous for generation of uniform fine recrystallized grains.

\section{Conclusion}

1. Herein, the effect of the deformation type on grain refinement was studied. The chip specimen generated smaller and considerably uniform recrystallized grains than the rolled specimen after subsequent heat treatment. This was attributed to the difference in the subgrain structure between cutting and rolling. The chip specimen's subgrains were smaller and more uniform than the subgrains of the rolled specimen, although the equivalent plastic strain of the chip specimen was smaller than the rolled specimen's subgrains. The causes of the variation in subgrain structure between the chip and rolled specimens were studied using three mechanisms.

2. Deformation boundary conditions of crystal grains in the work material were different because of the difference in the size of the plastic deformation zone between the cutting and rolling. In the case of cutting, grains were deformed in a thin shear plane that was thinner than the average grain diameter. When the grains passed through the shear plane, they deformed evenly, and they are subdivided into uniform ultrafine grains by cutting. However, the deformation zone in rolling was larger than the average grain diameter. The grains in the rolled material were rotated individually to a certain crystal orientation that enabled them to be easily deformed, and GNDs were accumulated on the grain boundaries. Therefore, the plastic strain in the grains was relaxed, and the subgrain generation was suppressed.

3. The subgrain generation was influenced by a high strain rate and cutting temperature.

4. The variation in shear and plane strain deformation influenced the development of the subgrain structure. The numerical simulation based on the crystal plasticity theory was used to study the effects of deformation type on subgrain refinement. More than 10 systems in each slip system family were activated during shear deformation, whereas less than nine systems were activated by plane strain compression. More slip systems were activated in cutting than that in the rolling process, and multislip systems moved regardless of the initial orientation. Misorientations occurred inside a grain, and GNBs were constructed. Thus, grains were subdivided into ultrafine grains with large misorientation boundaries. Because nucleation and grain growth occurred from these subgrains and shear deformation was effective for generation of uniform ultrafine recrystallized grains. Therefore, cutting was more efficient than rolling in producing ultrafine-grained steel.

\section{Appendix 1}

It is known that temperature of a chip in cutting reaches some hundred degrees in Celsius, and significant temperature difference occurs between the both surfaces of a chip. Appendix Fig. 9 shows an analytical model for calculation of temperature distribution change in a chip. Thickness of the strip is L, and the strip is continuous to vertical direction in the figure. At the beginning, temperature on 
the right surface $\left(x=\frac{L}{2}\right)$ is high, and temperature on the left surface $\left(x=-\frac{L}{2}\right)$ is low $\left(T_{2}>T_{1}\right)$. It is assumed that both surfaces are adiabatic, and temperature distribution is uniform to the vertical direction. The temperature change of this model is calculated using the one-dimensional heat conduction equation in the $\mathrm{x}$ direction.

$\frac{\partial T}{\partial t}=\alpha \frac{\partial^{2} T}{\partial x^{2}}$

where $\alpha$ is the temperature conductivity, which is obtained by the following equation

$\alpha=\frac{\kappa}{c \rho}$

$\kappa$ is the thermal conductivity, $\mathrm{c}$ is the specific heat, and $\rho$ is the density.

Solving this equation based on the adiabatic conditions and the initial temperatures $T_{1}$ and $T_{2}$ at the left and right surfaces $(x= \pm L / 2)$, the following function is obtained.

$T(x, t)=\sum_{-\infty}^{\infty} A_{n} e^{-\alpha p_{n}{ }^{2} t} \sin \left\{\frac{2 \pi}{L}\left(\frac{1}{4}+n\right) x\right\}+\frac{T_{2}-T_{1}}{2}$

Here, $A_{n}$ is determined from the initial temperature distribution. The term $e^{-\alpha p_{n}{ }^{2} t}$ in this equation represents the reduction of temperature distribution with the increase of time t. It is found from this equation that the temperature distribution in a chip decreases to $1 / 100$ within $0.0019 \mathrm{~s}$ as show by the following calculation. $t=\frac{\ln 100}{\alpha\left\{\frac{2 \pi}{L}\left(\frac{1}{4}+n\right)\right\}^{2}}=0.0019 \mathrm{sec}$

where $\mathrm{n}=0$ was assumed, and the material properties of pure iron and chip thickness $\left(\mathrm{t}_{2}=\mathrm{L}=0.15 \mathrm{~mm}\right)$ were used.

\section{Appendix 2}

Based on the theory of crystal plasticity, the strain rate $\varepsilon^{\circ}$ and spin tensor $\mathrm{W}$ of a square lattice single crystal are expressed using the following equations. Here, the unit cell of a crystal is considered as the coordinate system. In the following equations, a normal font represents a scalar, and a bold font represents a tensor or a vector.

$\dot{\varepsilon}=\sum_{k} p_{k} \dot{\gamma}_{k}$,

$\boldsymbol{W}=\sum_{k} \boldsymbol{\omega}_{k} \dot{\gamma}_{k}$

where $k$ is the slip system number, $\dot{\gamma}_{k}$ is the amount of slip deformation of each slip system and $\mathrm{p}_{k}$ and $\omega_{k}$ are tensors determined by the crystal's slip system as expressed by the following equation.

$\boldsymbol{p}_{k}=\frac{1}{2}\left(\boldsymbol{n}_{k} \otimes \boldsymbol{b}_{k}+\boldsymbol{b}_{k} \otimes \boldsymbol{n}_{k}\right)$

$\boldsymbol{\omega}_{k}=\frac{1}{2}\left(\boldsymbol{n}_{k} \otimes \boldsymbol{b}_{k}-\boldsymbol{b}_{k} \otimes \boldsymbol{n}_{k}\right)$
Fig. 9 Analytical model for calculation of temperature distribution change in a chip




$\mathrm{n}_{k}$ is the normal vector of the slip plane, and $\mathbf{b}_{k}$ is the Burgers vector of the slip system. $\otimes$ represents the tensor product.

When the stress $\sigma$ acts on the crystal, the resolved shear stress $\tau_{k}$ acting on the slip system $k$ is expressed by the following equation.

$\tau_{k}=p_{k} \bullet \boldsymbol{\sigma}$

Furthermore, to approximate the Schmidt law, which determines the active slip systems, the Asaro's constitutive equation is used, in which the shear strain rate $\dot{\gamma}_{k}$ on a slip system depends on the resolved shear stress $\tau_{k}$.

$\dot{\gamma}_{k}=\dot{\gamma}_{0} \operatorname{sgn}\left(\tau_{k}\right)\left|\frac{\tau_{k}}{\tau_{0}}\right|^{\frac{1}{m}}$

where $\tau_{0}$ is the reference shear stress, $\dot{\gamma}_{0}$ is the reference shear strain rate and $m$ is the shear rate dependence index. Furthermore, work hardening is assumed to occur due to deformation, and $\tau_{0}$ gradually increases.

The crystal plastic constitutive equation that expresses the stress-strain relationship is expressed as

$\dot{\sigma}=\boldsymbol{C}: \dot{\varepsilon}-\sum_{k} S_{k} \dot{\gamma}_{k}$

$\boldsymbol{S}_{k}=\left(\boldsymbol{\omega}_{k} \cdot \boldsymbol{\sigma}-\boldsymbol{\sigma} \cdot \boldsymbol{\omega}_{k}\right)+\boldsymbol{C}: \boldsymbol{p}_{k}$

where $\sigma$ represents the Jaumann velocity of the stress $\sigma$. C is an elastic modulus tensor.

The above equations are defined on the crystal lattice coordinate system. The stress rate and strain rate on the specimen coordinate system is calculated using

$\sigma=R \Sigma R^{T}$

$\dot{\varepsilon}=R \dot{E} R^{T}$

By substituting them into eqs. (A2.7) and (A2.8), the constitutive equation on the specimen coordinate system is obtained. $\mathrm{R}$ is a coordinate transformation tensor.

$\mathrm{R}$ gradually changes with the crystal rotation. The rate of change is expressed by the following equation using spin tensor

W which represents the change in crystal orientation.

$\dot{\boldsymbol{R}}=\boldsymbol{W} \boldsymbol{R}$

The coordinate transformation tensor $\mathrm{R}$ is updated using

$\boldsymbol{R}_{t+d t}=\boldsymbol{R}_{t}+\dot{\boldsymbol{R}} d t=\boldsymbol{R}_{t}+\boldsymbol{W} \boldsymbol{R}_{t} d t$

Internal stress and strain are uniforms in a cubic specimen made of a single crystal. Here, a pure iron bcc lattice is adopted and a slip system of $\{110\}\langle\overline{1} 11\rangle$ and $\{112\}\langle 11 \overline{1}\rangle$ is used as the active slip system. The initial crystal orientation is randomly selected to ensure that the existence probabilities are even in all orientations. Plane compressive strain or simple shear strain was used for the outside of the cubic specimen. The stress and strain rate on the crystal coordinate system are obtained from (A2.10) and (A2.9). Eq. (A2.3) is used to calculate $\mathrm{p}_{k}$, eq. (A2.4) is used to calculate $\omega_{k}$, and eq. (A2.5) and (A2.6) are used to determine the slip deformation rate (shear strain rate) $\dot{\gamma}_{k}$ of each slip system. The stress rate is calculated using eq. (A2.8) and (A2.7). The stress and strain after a time increment $d t$ is obtained and converted into the stress and strain on the specimen coordinate system. The spin tensor is determined, and the crystal orientation after a time increment $d t$ is determined. The above calculation procedure is repeated until a predetermined strain is achieved, and the final crystal orientation is obtained.

Acknowledgments Financial support from the Amada Foundation (AF-201910-B2) is acknowledged. The authors would like to thank Enago (www.enago.jp) for the English language review.

Funding This research was funded by the AMADA FOUNDATION, grant number AF-201910-B2.

\section{Declarations}

Conflict of interest The authors declare no conflict of interest.

Open Access This article is licensed under a Creative Commons Attribution 4.0 International License, which permits use, sharing, adaptation, distribution and reproduction in any medium or format, as long as you give appropriate credit to the original author(s) and the source, provide a link to the Creative Commons licence, and indicate if changes were made. The images or other third party material in this article are included in the article's Creative Commons licence, unless indicated otherwise in a credit line to the material. If material is not included in the article's Creative Commons licence and your intended use is not permitted by statutory regulation or exceeds the permitted use, you will need to obtain permission directly from the copyright holder. To view a copy of this licence, visit http://creativecommons.org/licenses/by/4.0/.

\section{References}

1. Mack MJ (2001) Minimally invasive and robotic surgery. JAMA 285:568-572. https://doi.org/10.1001/jama.285.5.568

2. Repici A, Arezzo A, De Caro G et al (2009) Clinical experience with a new endoscopic over-the-scope clip system for use in the GI tract. Dig Liver Dis 41:406-410. https://doi.org/10.1016/j. dld.2008.09.002

3. Dornfeld D, Min S, Takeuchi Y (2006) Recent advances in mechanical micromachining. CIRP Ann 55:745-768. https:// doi.org/10.1016/j.cirp.2006.10.006 
4. Schneider F, Das J, Kirsch B et al (2019) Sustainability in ultra precision and Micro machining: a review. Int J Precis Eng Manuf Technol 6:601-610. https://doi.org/10.1007/ s40684-019-00035-2

5. Komatsu T, Matsumura T, Torizuka S (2011) Effect of grain size in stainless steel on cutting performance in micro-scale cutting. Int J Autom Technol 5:334-341. https://doi.org/10.20965/ijat.2011. p0334

6. Komatsu T, Kobayashi H, Torizuka S, Nagayama S-i (2012) Micro hole piercing for ultra fine grained steel. Int J Autom Technol 6:802-808. https://doi.org/10.20965/ijat.2012.p0802

7. Komatsu T (2015) Effects of grain size on the groove depths in microlaser cutting of austenitic stainless steel SUS304. Int J Autom Technol 9:636-645. https://doi.org/10.20965/ijat.2015. p0636

8. Masumura RA, Hazzledine PM, Pande CS (1998) Yield stress of fine grained materials. Acta Mater 46:4527-4534. https://doi.org/ 10.1016/S1359-6454(98)00150-5

9. Masuda T, Fujimitsu K, Takizawa Y, Horita Z (2015) Achieving superplasticity through grain refinement of A2024 alloy round bar using high-pressure sliding. J Japan Inst Light Met 65:319-325. https://doi.org/10.2464/jilm.65.319

10. Hasegawa M, Osawa M (1984) Corrosion behavior of ultrafine grained austenitic stainless steel. Corrosion 40:371-374. https:// doi.org/10.5006/1.3593940

11. Torizuka S, Muramatsu E, Komatsu T, Nagayama S (2011) Production processes for nanostructured wires, bars and strips. In: Whang SH (ed) Nanostructured metals and alloys: processing, Microstructure, Mechanical Properties and Applications. Woodhead Publ Ltd, pp 715-746

12. Tsuji N (2002) Ultrafine grained steels. Tetsu-to-Hagane 88:359369. https://doi.org/10.2355/tetsutohagane1955.88.7_359

13. Azushima A, Kopp R, Korhonen A et al (2008) Severe plastic deformation (SPD) processes for metals. Cirp Ann Technol 57:716-735. https://doi.org/10.1016/j.cirp.2008.09.005

14. Segal VM, Reznikov VI, Drobyshevskiy AE, Kopylov VI (1981) Plastic working of metals by simple shear. Russ Metall:99-105

15. Segal VM (1995) Materials processing by simple shear. Mater Sci Eng A 197:157-164. https://doi.org/10.1016/0921-5093(95) 09705-8

16. Bridgman PW (1935) Effects of high shearing stress combined with high hydrostatic pressure. Phys Rev 48:825-847. https://doi. org/10.1103/PhysRev.48.825

17. Erbel S (1979) Mechanical properties and structure of extremely strainhardened copper. Met Technol 6:482-486. https://doi.org/ 10.1179/030716979803276363

18. Saito Y, Tsuji N, Utsunomiya H et al (1998) Ultra-fine grained bulk aluminum produced by accumulative roll-bonding (ARB) process. Scr Mater 39:1221-1227. https://doi.org/10.1016/S13596462(98)00302-9

19. Nagashima F, Nakagawa Y, Yoshino M (2020) Study on effects of strong shear strain on recrystallized grain size of pure iron and microstructure control method. Procedia Manuf 50:248-252. https://doi.org/10.1016/j.promfg.2020.08.046

20. Nagashima F, Nakagawa Y, Yoshino M (2020) Influence of severe plastic deformation on static recrystallization microstructure of pure Iron. Metals 10(10):1320

21. Barraclough DR, Sellars CM (1979) Static recrystallization and restoration after hot deformation of type 304 stainless steel. Met Sci 13:257-268. https://doi.org/10.1179/msc.1979.13.3-4.257
22. Cuddy LJ (1981) Microstructures developed during thermomechanical treatment of HSLA steels. Metall Trans A 12:1313-1320. https://doi.org/10.1007/BF02642345

23. Salvatori I, Inoue T, Nagai K (2002) Ultrafine grain structure through dynamic recrystallization for type 304 stainless steel. ISIJ Int 42:744-750. https://doi.org/10.2355/isijinternational.42.744

24. Baumeister T (1967) Standard handbook for mechanical engineers, 7th editio. McGraw-Hill, New York

25. Wronski S, Tarasiuk J, Bacroix B et al (2013) Microstructure heterogeneity after the ECAP process and its influence on recrystallization in aluminium. Mater Charact 78:60-68. https://doi.org/ 10.1016/j.matchar.2013.01.010

26. Dupin E, Yanagida A, Yanagimoto J (2014) Modeling static and dynamic kinetics of microstructure evolution in type 316 stainless steel. Steel Res Int 85:1099-1108. https://doi.org/10.1002/srin. 201300173

27. Usui E (1971) Sessaku/Kensakukakougaku. Kyoritsu Shuppan Co., Ltd., Tokyo

28. Kaibyshev R, Goloborodko A, Musin F et al (2002) The role of grain boundary sliding in microstructural evolution during superplastic deformation of a 7055 aluminum alloy. Mater Trans 43:2408-2414. https://doi.org/10.2320/matertrans.43.2408

29. Kamijo T (1968) On the development of the rolling textures in $<\mathrm{I}>$ fcc $<$ II $>$ metals. J Japan Inst Met 32:22-28. https://doi.org/ 10.2320/jinstmet1952.32.1_22

30. Engler O, Crumbach M, Li S (2005) Alloy-dependent rolling texture simulation of aluminium alloys with a grain-interaction model. Acta Mater 53:2241-2257. https://doi.org/10.1016/j.actam at.2005.01.032

31. Childs THC, Maekawa K, Obikawa T, Yamane Y (n.d.) Metal Machining, Theory and applications, ARNOLD, pp. 226-264

32. Hao G, Liu Z (2020) The heat partition into cutting tool at toolchip contact interface during cutting process: a review. Int J Adv Manuf Technol 108:393-411

33. Asaro RJ (1983) Crystal plasticity. J Appl Mech 50:921-934. https://doi.org/10.1115/1.3167205

34. Peirce D, Asaro RJ, Needleman A (1982) An analysis of nonuniform and localized deformation in ductile single crystals. Acta Metall 30:1087-1119. https://doi.org/10.1016/0001-6160(82) 90005-0

35. Kocks UF, Mecking H (2003) Physics and phenomenology of strain hardening: the FCC case. Prog Mater Sci 48:171-273. https://doi.org/10.1016/S0079-6425(02)00003-8

36. Groh S, Marin EB, Horstemeyer MF, Zbib HM (2009) Multiscale modeling of the plasticity in an aluminum single crystal. Int $\mathbf{J}$ Plast 25:1456-1473. https://doi.org/10.1016/j.ijplas.2008.11.003

37. Luo ZC, Yoshino M, Terano M, Yamanaka A (2018) Prediction of static recrystallization nucleation sites in tensile deformed single crystal pure Iron through a combination of in-situ EBSD and CPFEM. Metals (Basel) 8:15. https://doi.org/10.3390/met8100858

38. Lord AEJ, Beshers DN (1965) Elastic stiffness coefficients of Iron from $77^{\circ}$ to $673^{\circ} \mathrm{K}$. J Appl Phys 36:1620-1623. https://doi.org/10. $1063 / 1.1703098$

Publisher's note Springer Nature remains neutral with regard to jurisdictional claims in published maps and institutional affiliations. 